\title{
Strategies to Improve Detection of Hypertension Genes
}

\author{
Steven C. Hunt \\ Cardiovascular Genetics Division, Department of Internal Medicine, University of Utah, Salt Lake City, Utah, USA
}

The identification of genes that are associated with hypertension has been a slower process than for other diseases, despite a similar heritability in many cases. This is, in part, likely to be a result of a great number of genes being involved with the blood pressure control pathways and their accompanying small effects on the phenotypes that are being measured.

With blood pressure fluctuating so acutely with changes in posture, stress, activity level, and even while talking, strong responses to these fluctuations are required to keep blood pressure appropriate for tissue perfusion and cellular function. Because of the importance of blood pressure control, there are redundant compensatory pathways for this pressure normalization. Therefore, a gene that may compromise one pathway may not be found to be associated with hypertension because other pathways can adequately compensate and normalize the phenotypes being studied. What might be detected in these situations are associations of a gene with the compensating factors that change to normalize a causal pathway. The altered levels of the compensating factors will likely be smaller than the causal phenotype levels if there were no compensation, and are probably more difficult to detect. Both initiating genes and compensating genes are involved in eventual hypertension development, in line with the strong polygenic nature of hypertension.

Recent genome-wide association studies (GWAS) have begun to suggest genes related to hypertension and blood pressure levels [1-9]. Comparing the results of each GWAS as a whole, there is little overlap of the significant gene associations. When targeted in silico look-ups of association regions are done in other GWAS, a few genes are then replicated because the penalty for multiple corrections is decreased. These few genes, such as $\mathrm{SH} 2 \mathrm{~B} 3$ or $\mathrm{CACNB}$, show very small influences on blood pressure in accordance with the theory of large numbers of genes working together in pathways for fine blood pressure control. Interestingly, few of the physiological candidate genes that have been found associated with hypertension over the last 20 years of research have been replicated by GWAS. 
In light of these difficulties, elucidation of a few concepts suggested by the literature may increase the ability to detect more genes and to verify the genes already suggested for their involvement with hypertension. These include: (1) selecting of subjects with strong family histories of hypertension; (2) identifying age ranges that show the highest heritability of blood pressure in the target population; (3) measuring local tissue-specific electrolyte or hormone concentrations in addition to systemic concentrations; (4) using acute interventions to investigate genetic interactions with environmental factors such as diet, activity and stress; (5) determining the appropriate time window after intervention to identify initiators versus compensators of blood pressure elevation; (6) genotyping subjects involved in large clinical trials to detect intervention interactions with genotype, and (7) increasing the density of SNPs in and around candidate genes for hypertension in genome-wide or candidate gene association studies.

\section{Subject Selection}

Persons with strong family histories of hypertension, still the strongest genetic risk factor we have to predict future hypertension $[10,11]$, are more likely to have multiple genes predisposing to blood pressure elevation. Not only are these persons likely to have more hypertension genes, the genes shared among family members are sufficiently expressed to lead to hypertension in multiple family members - in genetic terms they are penetrant genes. Unaffected relatives in families with strong histories of hypertension have significantly higher risks of developing hypertension compared to the general population $[10,12]$. Relative risks above 2 are almost always seen in a positive family history of hypertension compared to relative risks for a specific gene in large hypertension genome-wide association studies in the 1.1-1.5 range. From birth to young adulthood, the combined effects of these genes seem to be adequately compensated, as hypertension does not generally develop until ages 40 or older. Aging is an extremely strong risk factor for hypertension, with the lifetime risk of hypertension approaching 90\%, as reported in the Framingham Heart Study [13]. This result suggests that compensating mechanisms may lose their effectiveness with age allowing the effects of initiating hypertension genes to be detected.

While a proportion of the non-genetic variance in blood pressure is due to measurement errors and random variation, a significant part is the result of environmental factors. Selection of hypertensive subjects who have more environmental risk factors as opposed to stronger genetic predispositions may lead to difficulties in detecting genes with small effects even though the combination of genetic and environmental risk factors may increase the risk of hypertension. On the other hand, selection of high-risk subjects and modulation of environmental risk factors should allow detailed investigation of gene and environment interactions and will likely improve the ability to detect hypertension genes. 


\section{Selecting an Intervention}

In the detection of initiating factors, one must correctly choose an intervention that affects the initiating genes by increasing or decreasing expression. One obvious choice would be dietary salt manipulation or saline infusions. Since $40-50 \%$ of the population appear to have blood pressures that are quite responsive to salt, this has been the most frequent intervention. It is still unclear whether sodium changes from low to high or high to low are best to unmask sodium excretion abnormalities. A feeding study in Japan showed differences in sodium excretion between salt-sensitive and salt-resistant subjects when changed from a low to high salt diet, but not vice versa [14]. However, an earlier study found that compared to salt-resistant patients, salt-sensitive patients had blunted renal responses when sodium intake was reduced rather than increased [15]. Saline infusions have been effective in validating the association of the alpha adducin gene with hypertension [16]. Weight loss interventions would be expected to affect the salt sensitivity pathways, as obese subjects are more likely to be salt sensitive, and salt sensitivity is decreased following weight loss [17]. Other interventions include potassium supplementation, angiotensin II infusions, antihypertensive medication institution, and stressed blood pressure procedures such as posture change, isometric handgrip procedures, exercise and mental challenges.

\section{Study Time Windows}

Animal models suggest that when the blood pressure system is stressed with high salt intake, an initial genetic response may be only transiently expressed. Compensatory mechanisms quickly counteract the abnormal genetic response to the initial stressor, reducing the blood pressure back to normal or near-normal levels. The Japanese salt study referenced above [14], showed that the sodium excretion differences were maintained between salt-sensitive and salt-resistant subjects only for a few days after the intervention, after which the excretion curves became similar again. Investigating the causes of the delayed sodium excretion would be effective during that window but not before or after.

Figure 1 shows a genetic cause of hypertension, the knockout of the microsomal prostaglandin E synthase-1 gene in the mouse [18]. Chronic salt loading acts as an initiator of sodium retention in the knockout mouse during days 1-3. This sodium retention is subsequently hidden or normalized after compensating mechanisms increasing sodium excretion are activated, even when sodium loading continues. Measuring sodium balance after day 3 in the 2 mouse strains would not identify this gene as being associated with sodium balance. The compensatory factors that are invoked to normalize the sodium retention are blood pressure increases that lead to premature death in these mice. In this case, the blood pressure difference would be detected as a compensating factor or the result of a compensating factor, but in other 


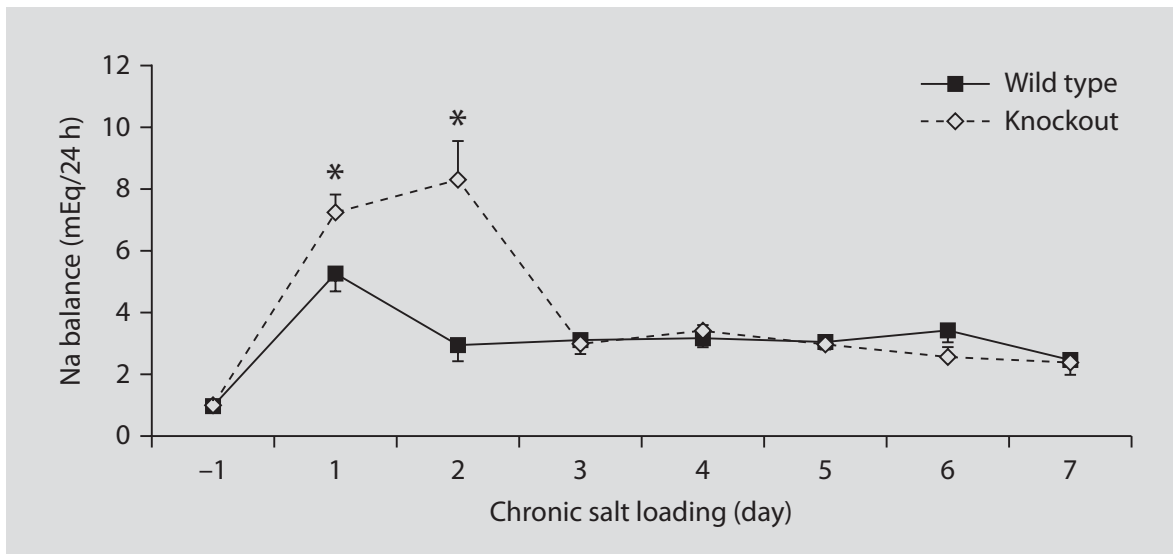

Fig. 1. Transient acute but not chronic sodium retention in the microsomal prostaglandin $E$ synthase-1 knockout (KO) mouse. From Jia et al. [18]. * $p<0.05$.

examples, the compensating factor might not be measured in the experiment, and it may not lead to elevated blood pressure. It seems likely that there may be time windows throughout life when specific genetic factors have greater expression and contribute to the development of hypertension.

The existence of these time windows is further suggested by the low infant twin blood pressure heritability [19]. Adult twin blood pressure heritability is much larger [20-22] and suggests that heritability is not constant over age [23-26]. Results from the HyperGEN study, fitting both the traditional constant heritability model and a model that allows heritability to change with age (fig. 2), suggests that blood pressure heritability is much larger in particular age ranges [26]. The maximum heritability also differs by racial group, suggesting that different populations may have different heritability patterns. For systolic blood pressure, blacks had an estimated peak heritability of 0.68 at age 59 compared to an average heritability of 0.29 ; whites had an estimated peak heritability of 0.69 at age 74 compared to an average heritability of 0.24 . The differences between the peak and average heritability were highly significant $\left(\mathrm{p}<10^{-12}\right)$. If these results are verified in other populations, they suggest that in order to maximize the ability to detect hypertension genes, the population-specific age-dependent heritability should first be estimated and subjects selected within the maximum range.

\section{Tissue versus Central Phenotype Measurement}

The importance of the kidney in blood pressure control and hypertension development and tissue-specific gene expression was shown in elegant experiments in which the AT1R gene was knocked out either systemically or just in the kidney [27]. In mice 


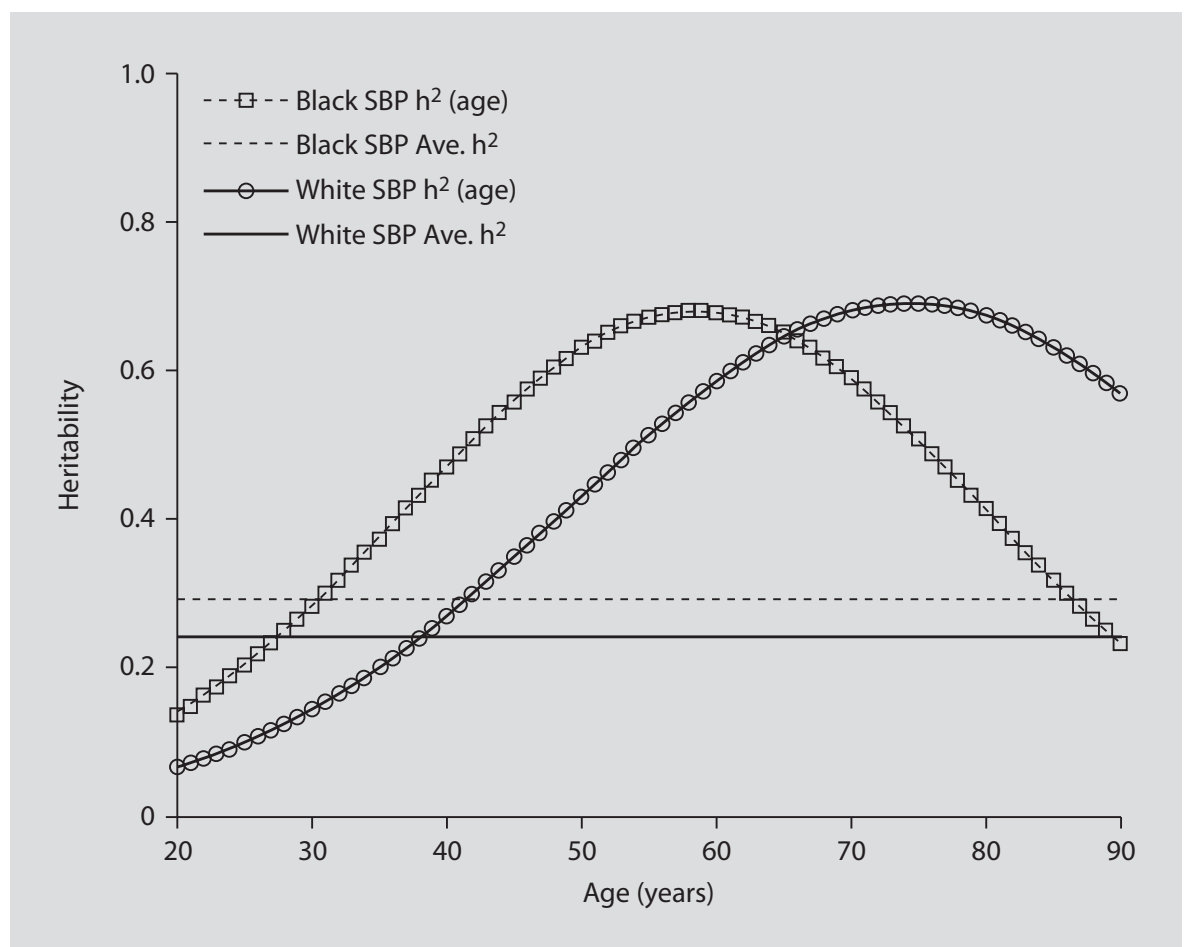

Fig. 2. Systolic blood pressure (SBP) average and age-specific heritabilities in white and black participants in the HyperGEN study. From Shi et al. [26].

where the AT1R gene was systemically knocked out but which then received a transplant of a normal kidney with functioning AT1 receptors, blood pressure increased and hypertension eventually developed during chronic angiotensin II infusion. When the AT1R gene was functional systemically and a kidney with the AT1R gene knocked out was transplanted into the mouse, hypertension did not develop during angiotensin II infusion. The study concluded that both central and kidney AT1 receptors help control blood pressure, but that the kidney receptors were required for the development of hypertension. These experiments also confirmed the earlier transplant experiments that showed that hypertension follows the kidney [28]. Kidneys transplanted from normotensive rats into hypertensive rats removed the hypertension and kidneys transplanted from hypertensive rats into normotensive rats caused hypertension. These experiments suggest that if polymorphims in kidney expressed genes such as the AT1R gene are associated with hypertension, one should measure the intermediate phenotypes in the kidney rather than systemically.

An example of such an intermediate phenotype is plasma angiotensinogen. Experiments on the relevance of circulating angiotensinogen versus angiotensinogen acting locally in the renal tubules show that kidney-specific angiotensinogen and the resulting angiotensin II produced play important roles in sodium excretion somewhat independently of circulating angiotensin II [29-31]. This reinforces the concept 
Table 1. Systolic and diastolic blood pressure reductions by angiotensinogen (AGT) genotypes after intervention in 3 clinical trials. From Hunt [40]

\begin{tabular}{lllll}
\hline & \multicolumn{4}{l}{ AGT G-6A } \\
\cline { 2 - 5 } & AA & GA & GG & AA-GG difference \\
\hline TOHP Na reduction & $-2.7 /-2.2$ & $-1.3 /-0.7$ & $-0.2 / 1.1$ & $-2.5 /-1.5$ \\
TOHP weight loss & $-3.5 /-2.4$ & $-0.9 /-1$ & $-1.1 / 0.3$ & $-2.6 /-1.4$ \\
SAGA salt diet & $-8.6 /-3.9$ & $-9.0 /-5.2$ & $-5.3 /-1.0$ & $-3.3 /-2.9$ \\
DASH fruit/vegetable diet & $-5.2 /-3.0$ & $-2.3 /-1.2$ & $-1.3 / 0.3$ & $-3.9 /-3.3$ \\
DASH diet & $-6.9 /-3.5$ & $-5.6 /-3.2$ & $-2.8 / 0.2$ & $-4.1 /-3.7$ \\
\hline
\end{tabular}

All blood pressure data are in mm Hg. TOHP = Trials of Hypertension Prevention II [33]; SAGA = Dutch clinical trial using SAGA salt [32]; DASH = Dietary Approaches to Stop Hypertension [34].

that the tissue in which hypertension risk factors are measured may be critical for detecting gene associations.

\section{Intervention Studies}

Some genes have been consistently related to elevated blood pressure and hypertension but the observed effects of these genes are small and therefore hard to replicate in all studies. The majority of the associated genes have been related to renal electrolyte handling, similar to mechanisms of the rarer monogenic hypertension disorders, possibly because this class of genes have been studied to a greater extent than genes not acting primarily in the kidney. The greater number of positive findings in the kidney-related genes may also be a result of the kidney genes having slightly larger effect sizes. The GWAS are beginning to find associations for a number of genes that are not specifically kidney related. Because there are many causes of hypertension, it would be expected that any one gene would only have a small effect when averaged across large numbers of people.

Intervention studies are one strategy that appear to magnify the baseline effects of genes so that they are more easily detected. Multiple interventions including reduced dietary salt, increased dietary potassium, increased fruits and vegetables, lower fat intake, weight loss, and drug treatment appear to help reduce blood pressure to a greater extent in subjects genetically susceptible to hypertension than in those not as susceptible. The results support the concept that those at highest genetic risk of hypertension compared to those at low risk show a greater improvement in blood pressure for interventions that target the defective genetic pathways.

Table 1 summarizes 3 clinical trials in which individuals at a greater risk of hypertension due to the $-6 \mathrm{~A}$ polymorphism in the AGT gene had the greatest reductions in blood pressure after intervention [32-34]. The Trials of Hypertension Prevention 
II enrolled subjects with borderline hypertension, the Dietary Approaches to Stop Hypertension (DASH) trial enrolled borderline or stage 1 hypertensive subjects, and the Dutch SAGA salt trial enrolled never-treated hypertensive subjects. All 3 trials showed a significant difference across AGT genotypes for change in blood pressure with the intervention. While sodium reduction was involved in 2 of the trials, similar results could be seen for weight loss [33], potassium supplementation [32] and the DASH diet even when sodium was held constant [34]. All interventions that target the genetically compromised pathway are likely to be effective in controlling blood pressure. Therefore, the $-6 \mathrm{~A}$ subjects on average appear to be salt sensitive and respond well to appropriate interventions despite their higher baseline risk for hypertension. Subjects with the AGT - $6 \mathrm{G}$ alleles would not be expected to respond as well and other interventions may be more effective.

The genetic effects of the -6A AGT allele on blood pressure can probably be easily confounded by other mechanisms. Obesity and the associated increase in adipocytes, which are an important source of circulating angiotensinogen, may have larger effects on blood pressure than the AGT gene in normal-weight subjects [35]. The combination of obesity with AGT should magnify the risks of hypertension beyond the additive effects of either one alone, even though it would be difficult to differentiate between the 2 risk factors. Higher LDL-C levels increase blood pressure responses to infused angiotensin II, making blood pressure more sensitive to angiotensin II even though the baseline blood pressure levels may not be elevated $[36,37]$. Greater blood pressure reactivity in the presence of high LDL-C may interact with stress and other environmental factors to eventually result in hypertension. Polymorphisms at the AT1 receptor also may affect the renin-angiotensin physiological pathway, with CC homozygotes at position A1166C of the AT1R gene showing smaller blood pressure responses to infused angiotensin II than A allele carriers [36].

\section{Improving Genome-Wide Association Results}

Genomic-wide association analyses of many common disease traits have been very successful at finding new genes [38]. Hypertension and blood pressure traits had had fewer genes suggested from these studies until very large meta analyses were performed that were powered sufficiently to detect very small genetic effects $[5,7]$. While these genes need further validation, it is noteworthy that few of the physiological candidate genes found to be associated with hypertension have been detected in the genome-wide scans. This suggests that other unknown but important genes for hypertension are also being missed. An important reason appears to be that the density of the SNPs on the arrays are not sufficient around these candidate genes [39]. Only 52\% of the HAPMAP SNPs in 160 hypertension candidate genes were captured by the Affymetrix 500k array. The greater the density of SNPs in a candidate gene, 
the greater the likelihood that an association can be found. More dense arrays are now available, but additional efforts should be made to target known candidate genes in these large studies. In addition, the less common SNPs should not be routinely excluded, as the sample size in these meta analyses may be sufficient to detect associations with SNPs with allele frequencies in the $0.5-5 \%$ range. Validated results from the genome-wide studies should be analyzed in existing clinical trials to test for intervention interactions, as has been done for AGT.

\section{Summary}

Multiple factors contribute to the development of hypertension, including genetic factors and environmental exposures. Various pathophysiological mechanisms are at play in the pathogenesis of hypertension and this pathogenesis, by necessity, exhibits substantial variation at the level of the individual, as it depends on the relative contribution of inherited genes and individual lifetime environmental exposures. Over time, long-term compensatory mechanisms, including responses to either chronic hypertension or to therapeutic intervention, can only obscure the initiating mechanisms of disease. Acute compensating mechanisms can also mask initiating gene effects during or after an intervention, so that early phenotype assessments during the intervention may be more likely to detect the genetic initiators. Compensatory mechanisms, working over days, weeks or even years, will likely be variably effective in minimizing the expected blood pressure rise, making it difficult to detect genetic initiating mechanisms in cross-sectional, 'steady state', or 'in balance' studies. If the lifetime risk of hypertension indeed approaches 90\% [13], the power to identify genetic factors can only decrease with duration of disease and treatment, and prediction of hypertension becomes of vanishing significance. With multiple factors at play, we cannot expect that all causes are mutually exclusive, but it is reasonable to assume that one of these mechanisms is predominant in the initiation of the disease in any one individual. Given the heterogeneity of essential hypertension argued above, it becomes evident that the chance of identifying genetic factors that contribute to disease development will be greatest if study subjects at highest genetic predisposition are observed during age ranges when heritability is at a maximum, using the correct phenotypes, measured in the correct tissues, during the correct time window. Genes found to be significant in such studies should be densely typed in clinical trials and large population studies to assess public health and clinical applications of the findings.

\section{Acknowledgments}

This work was supported by NIH grants AG18734 and HL090668. 


\section{Journal of
Nutrigenetics utrigenomics}

\begin{tabular}{l|l}
\hline J Nutrigenet Nutrigenomics 2010;3:182-191 \\
\hline $\begin{array}{l}\text { DOI: 10.1159/000324355 } \\
\text { Published online: April 6, 2011 }\end{array}$ & $\begin{array}{l}\text { @ 2011 S. Karger AG, Basel } \\
\text { www.karger.com/jnn }\end{array}$ \\
\hline
\end{tabular}

Hunt: Genetics of Human Hypertension

\section{References}

${ }_{1}$ Wellcome Trust Case Control Consortium: Genome-wide association study of 14,000 cases of seven common diseases and 3,000 shared controls. Nature 2007; 447:661-678.

-2 Levy D, Larson MG, Benjamin EJ, et al: Framingham Heart Study 100K Project: genome-wide associations for blood pressure and arterial stiffness. BMC Med Genet 2007;8(suppl 1):S3.

-3 Sabatti C, Service SK, Hartikainen AL, et al: Genome-wide association analysis of metabolic traits in a birth cohort from a founder population. Nat Genet 2009;41:35-46.

-4 Wang Y, O'Connell JR, McArdle PF, et al: Wholegenome association study identifies STK39 as a hypertension susceptibility gene. Proc Natl Acad Sci USA 2009;106:226-231.

5 Newton-Cheh C, Johnson T, Gateva V, et al: Genome-wide association study identifies eight loci associated with blood pressure. Nat Genet 2009;41: 666-676.

6 Org E, Eyheramendy S, Juhanson P, et al: Genomewide scan identifies $\mathrm{CDH} 13$ as a novel susceptibility locus contributing to blood pressure determination in two European populations. Hum Mol Genet 2009;18:2288-2296.

7 Levy D, Ehret GB, Rice K, et al: Genome-wide association study of blood pressure and hypertension. Nat Genet 2009;41:677-687.

$\$ 8$ Yang HC, Liang YJ, Wu YL, et al: Genome-wide association study of young-onset hypertension in the Han Chinese population of Taiwan. PLoS One 2009;4:e5459.

\$ Adeyemo A, Gerry N, Chen G, et al: A genomewide association study of hypertension and blood pressure in African Americans. PLoS Genet 2009;5: e1000564.

10 Hunt SC, Williams RR, Barlow GK: A comparison of positive family history definitions for defining risk of future disease. J Chron Dis 1986;39:809821.

-11 Hunt SC, Gwinn M, Adams TD: Family history assessment: strategies for prevention of cardiovascular disease. Am J Prev Med 2003;24:136-142.

12 Williams RR, Hunt SC, Heiss G, et al: Usefulness of cardiovascular family history data for populationbased preventive medicine and medical research (the Health Family Tree Study and the NHLBI Family Heart Study). Am J Cardiol 2001;87:129135.

13 Vasan RS, Beiser A, Seshadri S, et al. Residual lifetime risk for developing hypertension in middleaged women and men: The Framingham Heart Study. Jama. 2002 Feb 27;287(8):1003-10.
14 Sanada H, Yatabe J, Midorikawa S, et al: Singlenucleotide polymorphisms for diagnosis of salt-sensitive hypertension. Clin Chem 2006;52:352- 360.

$\checkmark 15$ Weinberger MH, Stegner JE, Fineberg NS: A comparison of two tests for the assessment of blood pressure responses to sodium. Am J Hypertens 1993; 6:179-184.

16 Manunta P, Cusi D, Barlassina C, et al: Alphaadducin polymorphisms and renal sodium handling in essential hypertensive patients. Kidney Int 1998; 53:1471-1478.

$\checkmark 17$ Rocchini AP, Key J, Bordie D, et al: The effect of weight loss on the sensitivity of blood pressure to sodium in obese adolescents. N Engl J Med 1989; 321:580-585.

-18 Jia Z, Zhang A, Zhang H, Dong Z, Yang T: Deletion of microsomal prostaglandin E synthase-1 increases sensitivity to salt loading and angiotensin II infusion. Circ Res 2006;99:1243-1251.

19 Levine RS, Hennekens CH, Perry A, et al: Genetic variance of blood pressure levels in infant twins. Am J Epidemiol 1982;116:759-764.

20 Feinleib M, Garrison RJ, Fabsitz R, et al: The NHLBI twin study of cardiovascular disease risk factors: methodology and summary of results. Am J Epidemiol 1977;106:284-295.

21 Miller JZ, Weinberger MH, Christian JC, Daugherty SA: Familial resemblance in the blood pressure response to sodium restriction. Am J Epidemiol 1987;126:822-830.

22 Hunt SC, Hasstedt SJ, Kuida H, et al: Genetic heritability and common environmental components of resting and stressed blood pressures, lipids, and body mass index in Utah pedigrees and twins. Am J Epidemiol 1989;129:625-638.

23 Vaughn TT, Pletscher LS, Peripato A, et al: Mapping quantitative trait loci for murine growth: a closer look at genetic architecture. Genet Res 1999;74:313322.

-24 Pérusse L, Moll PP, Sing CF: Evidence that a single gene with gender- and age-dependent effects influences systolic blood pressure determination in a population-based sample. Am J Hum Genet 1991; 49:94-105.

25 Cheng LS-C, Carmelli D, Hunt SC, Williams RR: Evidence for a major gene influencing 7-year increases in diastolic blood pressure with age. Am J Hum Genet 1995;57:1169-1177.

26 Shi G, Gu CC, Kraja AT, et al: Genetic effect on blood pressure is modulated by age: the Hypertension Genetic Epidemiology Network Study. Hypertension 2009;53:35-41. 


\section{Nutrigenetics \\ Nutrigenomics}

\begin{tabular}{l|l}
\hline J Nutrigenet Nutrigenomics 2010;3:182-191 \\
\hline $\begin{array}{l}\text { DOI: 10.1159/000324355 } \\
\text { Published online: April 6, 2011 }\end{array}$ & $\begin{array}{l}\text { ○ } 2011 \text { S. Karger AG, Basel } \\
\text { www.karger.com/jnn }\end{array}$ \\
\hline
\end{tabular}

Hunt: Genetics of Human Hypertension
27 Crowley SD, Gurley SB, Oliverio MI, et al: Distinct roles for the kidney and systemic tissues in blood pressure regulation by the renin-angiotensin system. J Clin Invest 2005;115:1092-1099.

28 Dahl LK, Heine M, Thompson K: Genetic influence of the kidneys on blood pressure: evidence from chronic renal homografts in rats with opposite predispositions to hypertension. Circ Res 1974;40:94101.

29 Rohrwasser A, Morgan T, Dillon HF, et al: Elements of a paracrine tubular renin-angiotensin system along the entire nephron. Hypertension 1999;34: 265-1274.

30 Navar LG, Nishiyama A: Why are angiotensin concentrations so high in the kidney? Curr Opin Nephrol Hypertens 2004;13:107-115.

- 31 Kobori H, Alper AB Jr, Shenava R, et al: Urinary angiotensinogen as a novel biomarker of the intrarenal renin-angiotensin system status in hypertensive patients. Hypertension 2009;53:344-350.

32 Hunt SC, Geleijnse JM, Wu LL, et al: Enhanced blood pressure response to mild sodium reduction in subjects with the $235 \mathrm{~T}$ variant of the angiotensinogen gene. Am J Hypertens 1999;12:460-466.

-33 Hunt SC, Cook NR, Oberman A, et al: Angiotensinogen genotype, sodium reduction, weight loss, and prevention of hypertension: trials of hypertension prevention, phase II. Hypertension 1998;32:393-401.
34 Svetkey LP, Moore TJ, Simons-Morton DG, et al: Angiotensinogen genotype and blood pressure response in the Dietary Approaches to Stop Hypertension (DASH) study. J Hypertens 2001;19: 1949-1956.

35 Janke J, Engeli S, Gorzelniak K, Luft FC, Sharma AM: Mature adipocytes inhibit in vitro differentiation of human preadipocytes via angiotensin type 1 receptors. Diabetes 2002;51:1699-1707.

36 Vuagnat A, Giacche M, Hopkins PN, et al: Blood pressure response to angiotensin II, low-density lipoprotein cholesterol and polymorphisms of the angiotensin II type 1 receptor gene in hypertensive sibling pairs. J Mol Med 2001;79:175-183.

37 Nickenig G, Sachinidis A, Michaelsen F, et al: Upregulation of vascular angiotensin II receptor gene expression by low-density lipoprotein in vascular smooth muscle cells. Circulation 1997;95:473478.

38 Manolio TA, Collins FS: The HapMap and genomewide association studies in diagnosis and therapy. Annu Rev Med 2009;60:443-456.

-39 Sober S, Org E, Kepp K, et al: Targeting 160 candidate genes for blood pressure regulation with a genome-wide genotyping array. PLoS One 2009; 4:e6034.

40 Hunt SC: Genetic architecture of complex traits predisposing to nephropathy: hypertension. Semin Nephrol, in press. 